\title{
Optical Trap Assisted Nanopatterning: Process Parallelization and Dynamic Structure Generation
}

\author{
Johannes Strauss $^{1,2}$, Marcus Baum ${ }^{1,2}$, Ilya Alexeev ${ }^{1,2}$, Michael Schmidt ${ }^{1,2}$ \\ ${ }^{* 1}$ Institute of Photonic Technologies, Friedrich-Alexander-Universität Erlangen-Nürnberg (FAU), \\ Konrad-Zuse-Straße 3/5, 91052 Erlangen, Germany \\ E-Mail: johannes.strauss@lpt.uni-erlangen.de \\ ${ }^{* 2}$ Erlangen Graduate School in Advanced Optical Technologies (SAOT), Friedrich-Alexander- \\ Universität Erlangen Nürnberg, Paul-Gordan-Straße 6, 91052 Erlangen, Germany
}

\begin{abstract}
In this publication we present a novel setup for the Optical Trap Assisted Nanopatterning (OTAN) technology. The setup allows process parallelization and thus higher throughput in this inventive and flexible direct-nanopatterning technology. We have determined the stiffness of the optical traps and compared the obtained result with the single beam OTAN parameters. Furthermore we estimate the increase in throughput for the parallelized approach in comparison to the conventional
\end{abstract} system.

DOI: 10.2961/jlmn.2017.02.0007

Keywords: nanostructuring, direct-write, optical trapping, ultrafast laser processing, holographic laser beam shaping

\section{Introduction}

Recently, submicron surface structuring applications have attracted considerable attention. Some examples of such structures can be found in micro sensors [1,2], nanostructured substrates for cell growth $[3,4]$ or even direct processing of cells $[5,6]$.

In this publication we present an extension of the direct laser write Optical Trap Assisted Nanostructuring (OTAN) process pioneered by Arnold and McLeod in 2008 [7] and investigated further in [8-11]. In the conventional OTAN approach a single transparent microsphere (most commonly either polystyrene or $\mathrm{SiO}_{2}$ with a diameter $0.5-10 \mu \mathrm{m}$ ) is trapped with the help of some optical tweezers and guided along the surface to be structured while being simultaneously irradiated with a second laser beam. The trapped sphere acts as a micro lens focusing the second, structuring laser beam onto the substrate's surface resulting in material modification or ablation. The main feature of such an approach is that it allows generating structures with characteristic dimensions smaller than the far field diffraction limit [7]. Additionally, being a direct write method, the technique allows generation of arbitrary surface patterns. Unfortunately, the serial structuring approach combined with low feed speed severely restricts the method's throughput. The low feed rate about $20 \mu \mathrm{m} / \mathrm{s}$ is needed to avoid losing the microsphere during the structuring due to the repulsion by the ablated material [11]. Tsai et al. [10] and McLeod et al. [12] demonstrated an approach for OTAN parallelization using a misaligned Mach-Zehnder interferometer setup, but it did not allows independent control of the structuring microspheres and imposed a strict limitation on the number of trap potentials.

In this contribution, we present a novel setup for OTAN structuring process parallelization that allows not only to significantly increase the number of trap potentials but also to independently control them. The presented setup is based on the use of Holographic Optical Tweezers (HOT) technique. Additionally, we demonstrate that a single laser can be used for the beads trapping and the structuring.

\section{Experimental setup}

It should be kept in mind that two types of structuring, schematically depicted in Fig. 1, must be clearly distinguished. In the first case called parallel structuring (Fig. 1a) all microspheres are tweezed at fixed laser traps and are capable of generating identical patterns only. For example, this can be achieved via relative movement of the beads with respect to the surface to be structured. In the case of simultaneous structure generation shown in Fig. 1b, each microsphere is independently controlled by the means of holographic beam shaping and simultaneous fabrication of different patterns is possible.

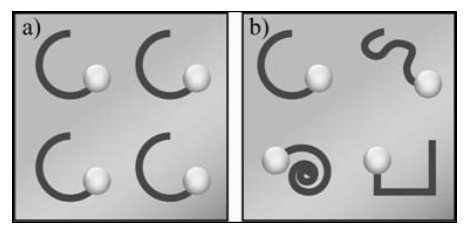

Fig. 1 a) parallel and b) simultaneous structure generation.

The experimental optical system is set up of various parts as shown in figure 2. They are listed group wise and in the sequence of the laser beam propagation.

The light source is a femtosecond (fs) laser: Origami $10 \mathrm{XP}$ from onefive $\mathrm{GmbH}$, Switzerland. It operates at a central wavelength of $1030 \mathrm{~nm}$ and a repetition rate of $1 \mathrm{MHz}$. The maximum pulse energy is $3.5 \mu \mathrm{J}$ and the pulse duration is $355 \mathrm{fs}$. The emitted laser beam is split into the trapping and the structuring arms. Power of each beam can be controlled independently. In order to avoid damage of the microspheres by the trapping beam (the beads are lo- 
cated in the foci of the trapping beam), the trapping laser pulse are temporarily elongated. The pulse elongation is achieved in a diffraction grating stretcher, similar to the one commonly used in the chirped pulse amplification technology [13].

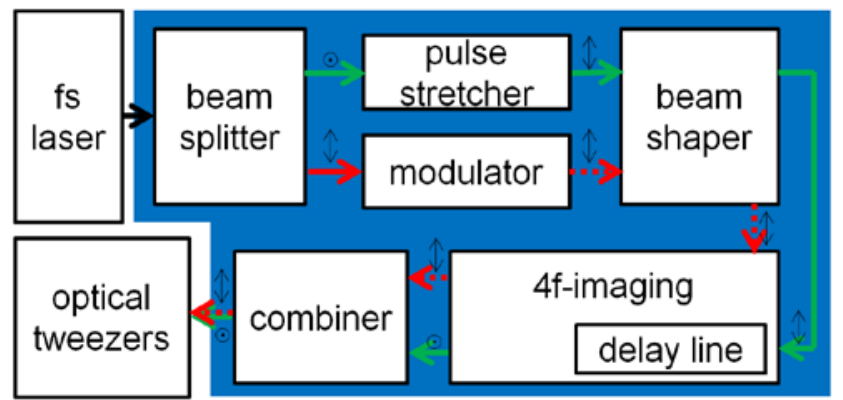

Fig. 2 Schematic layout of the optical design to parallelize the OTAN process. The blue box contains the components for system parallelization. The solid green line indicates the trapping and the dashed red line the structuring beam paths respectively. The small arrows and circles indicate horizontal and vertical polarization.

The trapping and the structuring beams are independently phase modulated using a spatial light modulator (SLM, Hamamatsu X10468). Here, the SLM active area is effectively split in two halves, with each one being used to independently control the corresponding laser beam. This beam shaper setup is controlled by custom-made LabVIEW software calculating the computer generated holograms using the Gerchberg-Saxton-Algorithm [14] with Fraunhofer propagation. To achieve real time control of microsphere positioning the calculations are performed with a graphics processing unit (Nvidia GTX 970). The optical tweezers are the last component in the experimental setup. Before, both beam paths are spatially combined by an inversely implemented polarizing beam splitter. The polarization direction of the trapping and structuring beams are orthogonal to each other. To ensure proper imaging of the SLMs subareas onto the entrance pupil of the microscopic lens (Nikon EPlan 100x, NA 1.25) in the tweezers, both beam paths must be identical in their length. This is achieved by incorporating a retroreflector based delay line into the trapping beam arm. The SLM imaging is implemented in the 4-f-configurtaion using a pair of identical lenses ( $f=400 \mathrm{~mm}$, Thorlabs, LA1172-B) at each beam path.

In order to increase the structuring area beyond the field of view of the microscopic lens, the sample to be processed is mounted on a $3 \mathrm{D}$ linear translation stage (Thorlabs MAX341/M). The parallel structuring as shown in Fig. 1a is achieved by stage movement only, while the simultaneous structure generation (Fig. 1b) is accomplished via independent movement of the microspheres. The latter is achieved by the SLM beam shaping. To monitor the structuring process the sample is illuminated with a broad band light source while the microscopic lens, an additional tube lens, and a digital camera (IDS UI-1220SE-M-GL) are arranged in the inverse bright field microscope configuration. A more detailed description of this optical tweezers subsystem can be found in [15].

\section{Results and discussion}

In order to ensure proper functioning of the experimental setup we had to determine the phase function of the SLM and verify its spatial homogeneity, measure the stretched pulse duration to establish the maximum applicable laser power that allows stable trapping of microspheres without damaging them. Finally we measured the trapping stiffness of the tweezers and demonstrated independent control of multiple beads.

\subsection{Phase response of the SLM}

When using a Spatial Light Modulator based on Liquid Cristal on Silicon (LCoS) to manipulate the phase of incident light, the phase function and the wrapping factor are the most important values to be measured for calibrating the system. The phase function describes the optical response of the display to the electric control signal. The wrapping factor is the bit value to which the calculated phase hologram must be scaled and wrapped when it is displayed in the SLM.

Recently published work by Engström et al. [16] shows measurements that underline the importance of this type of calibration for accurate control of the SLM induced phase shifts. Our calibration procedure is similar to the one described in Ref. [16] with the results plotted in Fig. 3

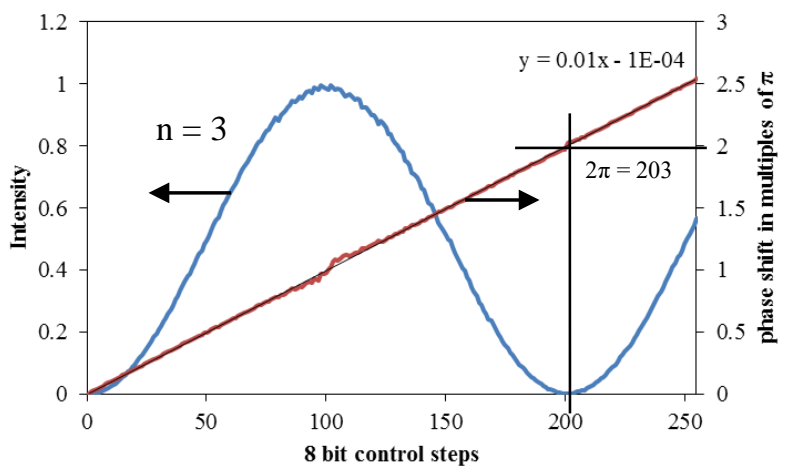

Fig. 3 Intensity (blue) and calculated phase shift (red) of the first diffraction order when a binary grating is applied at different step heights. The mean standard deviation is 0.0040 and 0.0054 , respectively.

Here, the active area of the SLM was completely overfilled by the laser beam at a wavelength of $1030 \mathrm{~nm}$. Then a binary grating with a periodicity of 20 Pixels and variable phase shift was displayed. For each control step height (0 to 255) the laser power in the first diffraction order was measured (blue, sinusoidal line). The data was normalized and the phase function was then calculated according to Equation (1). The red line represents the calculated phase function, which is linear fitted after that.

$$
\varphi^{\text {wrapped }}=2 \sin ^{-1}\left(\sqrt{p_{ \pm 1}^{\text {norm }}}\right) ; \quad p_{ \pm 1}^{\text {norm }} \in[0,1]
$$

After the first intensity maximum (blue, sinusoidal line) $2 \pi$ unwrapping must be implemented (2).

$$
\varphi= \begin{cases}\varphi^{\text {wrapped }}, & <\max \\ 2 \pi-\varphi^{\text {wrapped }}, & >\max \end{cases}
$$

Based on the measured diffraction efficiency it can be concluded that SLM phase response is constant over the entire SLM active areas. Otherwise it would have had been 
impossible to achieved the diffraction efficiency of 0 at the induced phase shift of $2 \pi$. The phase response is constant over the whole active area of the SLM. This was proven by spatially resolved measurements of the phase function and also can be directly seen in figure 3. If the SLM showed spatial deviations the intensity at $2 \pi$ would have not dropped to zero. The wrapping factor was determined at a bit value of 203, which slightly deviates from the value of 209 provided by the manufacturer. The linear phase function and the wrapping factor were subsequently incorporated into the control software.

\subsection{Power limits for trapping}

The stiffness of the trap is an important factor for the structuring accuracy in the OTAN process [17]. In the conventional setup operating with a continuous wave (cw) laser, the trap stiffness can be increased by increasing the laser power without risking to damage the structuring bead. In the case of the quasi cw ultrafast laser trapping, even relatively modest average laser power may results in the peak focal intensity above the damage threshold of the structuring bead.

Here the critical energy density that causes nonlinear absorption in the microsphere is easily reached [18] and thus limits the maximum average laser power that can be applied for trapping the particle.

To demonstrate the necessity of pulse stretching to obtain stable trapping the damage thresholds of the particle materials at $400 \mathrm{fs}$ and $40 \mathrm{ps}$ are compared. Typical materials of microspheres we use in OTAN are: polystyrene (PS), quartz glass $\left(\mathrm{SiO}_{2}\right)$, and sapphire $\left(\mathrm{Al}_{2} \mathrm{O}_{3}\right)$. Damage thresholds for these materials can be found in literature (Table 1 ).

Table 1 damage thresholds for microsphere materials

\begin{tabular}{cccc}
\hline $\begin{array}{c}\text { Damage } \\
\text { threshold } \Phi\end{array}$ & PS [19] & $\mathrm{SiO}_{2}[20]$ & $\mathrm{Al}_{2} \mathrm{O}_{3}[21]$ \\
\hline$\sim 400 \mathrm{fs}$ & $4.5 \mathrm{~J} / \mathrm{cm}^{2}$ & $2.1 \mathrm{~J} / \mathrm{cm}^{2}$ & $7.3 \mathrm{~J} / \mathrm{cm}^{2}$ \\
$\sim 40 \mathrm{ps}$ & $-/-$ & $8.1 \mathrm{~J} / \mathrm{cm}^{2}$ & $35 \mathrm{~J} / \mathrm{cm}^{2}$ \\
ratio & $-/-$ & 3.86 & 4.79
\end{tabular}

The ratio of the damage thresholds for the $40 \mathrm{ps}$ and $400 \mathrm{fs}$ pulses should be equivalent to the average power increase which can be used for trapping the microspheres. As the trapping stiffness increases linearly with the average laser power, pulse stretching into the ps-regime should improve performance of the optical tweezers. While comparison of the damage thresholds predicts that the trapping power can be increased only by a factor of 4 , we were able to apply significantly higher average laser powers, up to 1.2 W (limited by the laser) without observing any damage to the microspheres. Without the stretching the average laser power for trapping is limited to $40 \mathrm{~mW}$ only.

\subsection{Pulse duration}

To use a single laser source only for trapping particles and for structuring, an ultra-short pulsed laser system must be chosen. Ultra-short pulsed lasers provide sufficient fluence for multiphoton processes to create structures with OTAN technology. But when setting up a stable trap with a pulsed laser, high fluences will damage the particles before average power necessary for trapping is achieved, compare section 3.2. Therefore the pulse duration of the laser has to be increased to a value ensuring stable trapping. We decided to stretch the pulse by two orders of magnitude and chose the dispersion of our pulse stretching device accordingly.

The pulse duration of the original pulse and of the stretched pulse were measured. The results are shown in figure 4. Since it was not possible to measure the stretched pulse with our commercial autocorrelator (Pulse Scout by Spectra Physics), because of its restricted measurement range of 50-3500 fs, we had to build up our own device. The basis of the intensity autocorrelator is a Michelson interferometer with a fixed and a variable beam path. At the output both beam paths are overlapped coaxially and focused into a lithium niobate (LiNbO3) crystal to generate the second harmonic (SH). To measure the autocorrelation signal a $750 \mathrm{~nm}$ short pass filter was mounted in front of a calibrated photo diode to block the fundamental $(1030 \mathrm{~nm})$ so that only the $\mathrm{SH}$ is detected. By scanning of one interferometer arm the $\mathrm{SH}$-signal was acquired. As the $\mathrm{SH}$ signal scales quadratic with the fundamental, the square root of the measured date is calculated. Then full width at half maximum (FWHM) of the signal multiplied with a signal dependent deconvolution factor reveals the pulse duration.

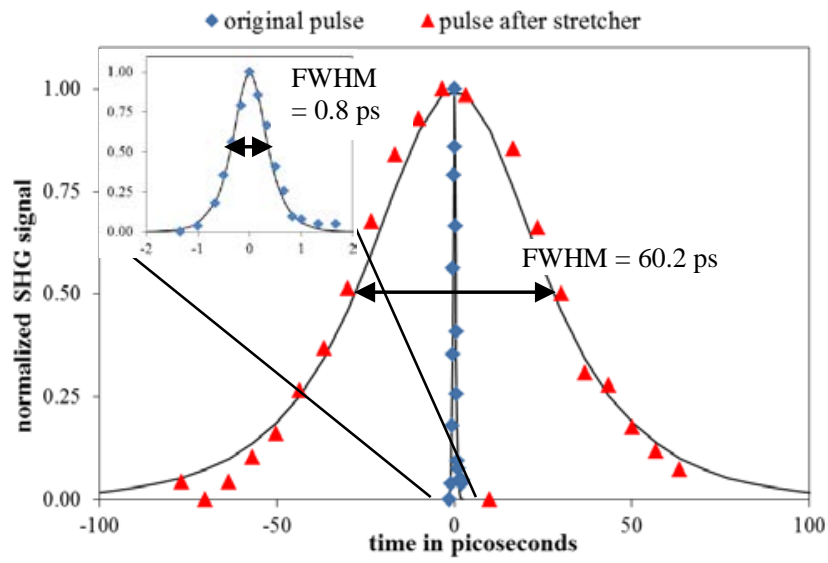

Fig. 4 Autocorrelation signal of the original and stretched pulse.

$$
\tau=D_{A C} * F W H M
$$

With the deconvolution factor $\mathrm{D}_{\mathrm{AC}}$ for a sech ${ }^{2}$ pulse: 0.567 [22]. We measured the pulse duration $\tau$ of the original pulse at $450 \mathrm{fs}$ and $34.1 \mathrm{ps}$ for the stretched pulse, respectively. This means that the pulse stretcher is elongation the laser pulse by a factor of 75. This result is in good agreement to the measured pulse duration of the original laser pulse at $480 \mathrm{fs}$, acquired with our commercial autocorrelator.

\subsection{Stability of multiple traps}

It has been shown that the optical trap stiffness strongly affects the accuracy of the OTAN structuring process [17]. While the structuring accuracy limit on the order of $5 \mathrm{~nm}$ has been predicted [17], the actual lateral jitter of the trapped microsphere becomes inversely proportional to the average laser power. For a $2.01 \mu \mathrm{m} \mathrm{SiO}_{2}$ bead trapped with $500 \mathrm{~mW}$ the averaged lateral position deviation has been measured to be $30 \mathrm{~nm}$. If the laser power is reduced to $150 \mathrm{~mW}$ the same position deviation is getting increased to 
$110 \mathrm{~nm}$. This uncertainty in the structuring bead positioning effectively defines the minimum average laser power needed for trapping.

As the stiffness of an optical trap cannot be measured directly a common approach is to measure the vibration of a trapped particle. In this measurement the particle's movement is detected by a fast quadrant detector (Thorlabs PDQ80A, bandwidth: $150 \mathrm{kHz}$ ). The experimental data is Fourier transformed and the power spectral density (PSD) is obtained. The experimental PSD data is fitted with a theoretically predicted Lorentzian curve expressed in equation 4, where D is the Einstein's coefficient of diffusion and f0 is the corner frequency. A typical PSD data set fitted with the Lorentzian curve is shown in Fig. 5. The corner frequency f0 extracted from the fit allows for quick evaluation of the trap stiffness [23] according to equation 5:

$$
\begin{aligned}
& P S D_{\text {fit }}=\frac{D}{2 \pi^{3}\left(f_{0}^{2}+f^{2}\right)} \\
& \kappa=12 \pi^{2} \cdot \eta \cdot a \cdot f_{0}
\end{aligned}
$$

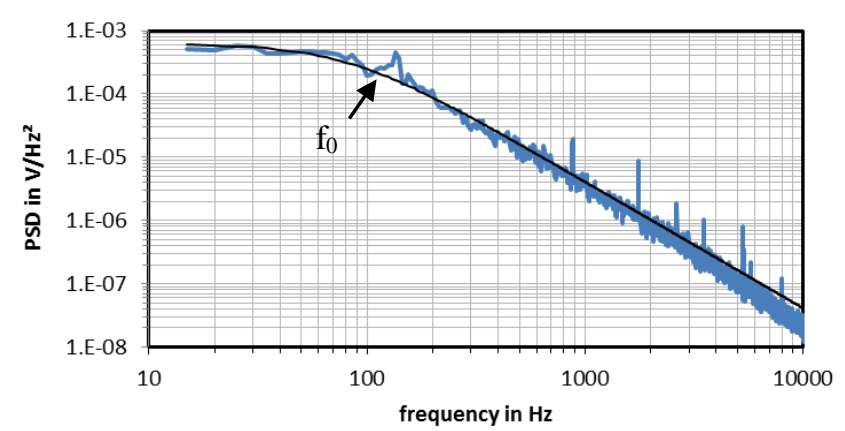

Fig. 5 PSD measurement plot and its Lorentzian fit for a $2.0 \mu \mathrm{m}$ $\mathrm{SiO}_{2}$ particle trapped with $100 \mathrm{~mW}$ average laser power

For trapping particles without beam shaping we determine a stiffness of $39.9 \mathrm{pN} / \mu \mathrm{m}$ with a corner frequency of 196.0 Hz, the viscosity of water $\eta=1.0$ at $20^{\circ} \mathrm{C}$ and the particles diameter $\mathrm{a}=2.0$. Here a $\mathrm{SiO}_{2}$ microsphere was trapped at the position of the zeroth diffraction order (red solid dot in figure 6). The average laser power was adjusted to $100 \mathrm{~mW}$, which is a typical value for experiments performed with cw trapping laser.

With the maximum average power of $1.2 \mathrm{~W}$ theoretically 12 particles can be trapped and moved simultaneously. We could demonstrate this by sequentially forming the letters $\mathrm{L}, \mathrm{P}$ and $\mathrm{T}$ with at least 11 particles, compare figure 6 .

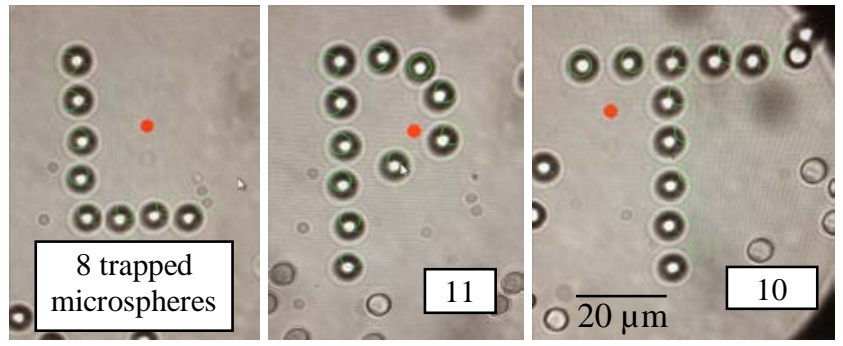

Fig. 6 Formation of the letters L, P and T with $5 \mu \mathrm{m}$ PS-beads by the use of HOT.

\section{Conclusion and outlook}

In this work we present a novel setup allowing for parallelization of the Optical Trap Assisted Nanopatterning process. This is achieved by the use of a pair of Holographic Optical Tweezers where the trapping beam and the structuring beam can be phase modulated separately. For the phase modulation one Spatial Light Modulator from Hamamatsu is incorporated into the system. We show that the phase function and the spatial uniformity of Hamamatsu's device are well calibrated in contrast to the results presented by Engström et al. They measured poor phase response and spatial deviant behavior of SLM devices. Only the wrapping factor of $2 \pi$ turned out to be different from the data delivered from the manufacturer.

Furthermore, we were able to reduce the amount of laser sources of our OTAN system, which now operates with one laser source only. We chose a fiber based femtosecond laser source as they are very stable in time and turn-key ready, which makes these systems interesting for industrial applications. We had to stretch the fs-pulses in time for successfully trapping microspheres. However, to finally proof the structuring capability we have to reduce the repetition rate of the structuring laser to ensure sufficient time for recapturing the microsphere when they are repulsed by the ablation. Nevertheless we expect an increase of the throughput by the amount of trapped particles. That means we can already expect a speed up the OTAN process by a factor of 11 based on our current results.

\section{Acknowledgements}

The authors gratefully acknowledge funding of the project within the priority program 1327 "Optisch erzeugte Sub-100-nm-Strukturen für biomedizinische und technische Applikationen” and funding of the Erlangen Graduate School in Advanced Optical Technologies (SAOT) both by the German National Science Foundation (DFG).

\section{References}

[1] A. Pimpin and W. Srituravanich: Engineering Journal; Vol 16 (2012): Regular Issue, (2011)

[2] B.-B. Xu, Y.-L. Zhang, H. Xia, W.-F. Dong, H. Ding and H.-B. Sun: Lab Chip, 13 (2013), 1677

[3] P. Roach, T. Parker, N. Gadegaard and M.R. Alexander: Surface Science Reports, 65 (2010), 145

[4] M. Licht, M. Straub, K. König, M. Afshar, D. Feili and H. Seidel, (2011), 79080M

[5] V. Kohli and A.Y. Elezzabi: WIREs Nanmed Nanobiotech, 1 (2009) 11

[6] E. Stratakis, A. Ranella, M. Farsari and C. Fotakis: Progress in Quantum Electronics, 33 (2009) 127

[7] E. McLeod and C. B. Arnold: Nature Nanotechnology 3 (2008) 413

[8] U. Quentin, B. Berg, I. Alexeev and M. Schmidt: Proceedings of LAMP (2013)

[9] K.-H. Leitz, Y.-C. Tsai, F. Flad, E. Schäffer, U. Quentin, I. Alexeev, R. Fardel, C. B. Arnold and M. Schmidt: Applied Physics Letters, 102 (2013) 243108

[10] Y.-C. Tsai K.-H. Leitz, R. Fardel, A. Otto, M. Schmidt and C. B. Arnold: Nanotechnology 23 (2012) 165304

[11] U. Quentin, K.-H. Leitz, I. Alexeev and M. Schmidt: Journal of Laser Micro/Nanoengineering, 7 (2012) 143 
[12]E. McLeod and C. B. Arnold: Opt. Exp. 17 (2009) 3640

[13]D. Strickland and G. Mourou: Opt. Commun. 56, (1985) 219

[14] R. W. Gerchberg and W. O. Saxton: Optik 35 (1972) 227

[15] U. Quentin, K.-H. Leitz, L. Deichmann, I. Alexeev and M. Schmidt: Journal of Laser Applications 24 (2012) 042003

[16]D. Engström, M. Persson and J. Bengtsson: Optics Express. 21 (2013) 16086

[17]U. Quentin, K.-H. Leitz, J. Paulus, I. Alexeev and M. Schmidt: Proceedings of LPM (2012)

[18] K.-B. Im, S.-B. Ju, S. Han, H. Park, B.-M. Kim, D. Jin and S.-K. Kim: Journal of the Korean Physical Society, 48 (2006) 968

[19] S. Joudkazis, K. Yamasaki, S. Matsuo and H. Misawa: Applied Physics Letters 84 (2004)

[20]B. C. Stuart, M. D. Feit, S. Herman, A. M. Rubenchik, B. W. Shore and M. D. Perry: Physical Review B 53 (1996)

[21]B. Bussière, O. Utéza, N. Sanner, M. Sentis, G. Riboulet, L. Vigroux, M. Commandré, F. Wagner, J.-Y. Natoli and J.-P. Chambaret: Applied Optics 51, (2012)

[22] P. Lazaridis, G. Debarge and P. Gallion: Opt. Lett. 20 (1995) 1160

[23] K. Berg-Sorensen and H. Flyvbjerg: Review of Scientific Instruments 75 (2004)

(Received: May 23, 2015, Accepted: July 30, 2017) 